Cahiers de philosophie de l'université de

CAHIERS DE PHILOSOPHIE
DE L LUNIVERSTIE DE CAEN

Caen

48 | 2011

Philosophie russe

\title{
Les noms. Éléments de réflexion sur l'onomatologie du père Pavel Florenskij
}

Florence Corrado-Kazanski

\section{OpenEdition}

1 Journals

Édition électronique

URL : https://journals.openedition.org/cpuc/961

DOI : $10.4000 /$ cpuc.961

ISSN : 2677-6529

Éditeur

Presses universitaires de Caen

Édition imprimée

Date de publication : 31 octobre 2011

Pagination : 63-74

ISBN : 978-2-84133-385-1

ISSN : $1282-6545$

Référence électronique

Florence Corrado-Kazanski, «Les noms. Éléments de réflexion sur l'onomatologie du père Pavel Florenskij », Cahiers de philosophie de l'université de Caen [En ligne], 48 | 2011, mis en ligne le 01 septembre 2020, consulté le 02 février 2023. URL : http://journals.openedition.org/cpuc/961 ; DOI: https://doi.org/10.4000/cpuc.961

\section{cc) (†) 8}

Creative Commons - Attribution - Pas d'Utilisation Commerciale 4.0 International - CC BY-NC 4.0

https://creativecommons.org/licenses/by-nc/4.0/ 


\section{Les noms. Éléments de réflexion sur l'onomatologie du père Pavel Florenskij}

\section{$\mathrm{L}$}

A QUESTION DU NOM peut être considérée comme une thématique récurrente de la pensée du père Pavel Florenskij. Dès ses études à l'Académie ecclésiastique de Moscou, il consacre plusieurs travaux à la question du changement de nom (pereimenovanie) dans la vie spirituelle: il compose ainsi, en 1906, Le changement de nom comme signe extérieur de changements dans la conscience religieuse ${ }^{1}$, et, en 1907, Du changement de nom sacramentel en lien avec la question du changement de nom de baptême dans la secte des beguny et des stranniki ${ }^{2}$, qui seront ensuite regroupés en un seul ouvrage Le changement de nom sacré3. Ce livre peut à son tour être considéré comme le fondement de son ouvrage Les noms (Imena), que le père Florenskij enrichira progressivement tout au long des années 1910, et qu'il terminera en 1923-1926.

Le point de départ de la réflexion du père Florenskij sur le nom est la question du lien entre le nom et la personne, comme l'indique le titre complet: Les noms. Remarques selon l'ordre alphabétique concernant l'onomatologie en tant que science des catégories de l'être personnel. Mais à partir de 1912-1913, en lien avec la dispute athonite concernant la nature du Nom de Dieu, le père Florenskij va approfondir l'étude philosophique et théologique de la question du nom, jusqu'à devenir l'un des théoriciens de la Glorification du Nom, ou onomatodoxie (imjaslavie). L'intérêt personnel du père Pavel Florenskij rencontre ainsi un événement historique, si bien que sa réflexion peut être considérée comme révélatrice de toute une

1. Izmenenie imen kak vnešnij znak peremen $v$ religioznom soznanii.

2. O sacramental'nom pereimenovanii v svjazi s voprosom o kreščal’nom pereimenovanii sekty begunov ili strannikov.

3. Svjaščennoe pereimenovanie. Pour toutes ces remarques historiques, l'auteur est entièrement redevable à l'introduction de l'higoumène Andronik à l'ouvrage Les noms (Imena) dans l'édition de référence des œuvres du père P. Florenskij, Sočinenija, Moskva, Mysl', 2000, 4 vol., t. 3(2), p. 523-528. 
atmosphère intellectuelle, alimentée à la fois par l'activité de philosophes, de théologiens, et de poètes. Nous évoquerons donc tout d'abord la question de l'onomatologie en général au début du $\mathrm{XX}^{\mathrm{e}}$ siècle en Russie, puis nous présenterons la méthode suivie par le père Florenskij dans son ouvrage Les noms, avant d'étudier les différentes définitions du nom qu'il donne, puis de proposer une lecture du commentaire que le père Florenskij fait de son propre nom, Pavel (Paul).

\section{La question de l'onomatologie au début du $\mathrm{XX}^{\mathrm{e}}$ siècle en Russie}

La publication en 1912 de l'ouvrage du moine Ilarion Sur les montagnes $d u$ Caucase $^{4}$, dans lequel il décrit son expérience spirituelle de la prière de Jésus ${ }^{5}$, constitue le point de départ d'une querelle autour du statut du Nom de Dieu, qui ébranle toute la communauté orthodoxe russe. La thèse de la glorification du Nom de Dieu apparaît comme le fruit d'une expérience mystique de la prière. Le mouvement onomatodoxe des moines du mont Athos affirme que dans la prière, Dieu lui-même est présent dans Son Nom, et que par conséquent, «le Nom de Dieu est Dieu lui-même » ${ }^{6}$. Le mouvement de la glorification du Nom va engendrer tout un débat théologique auquel prend part un grand nombre de penseurs et philosophes. La querelle des onomatodoxes et des onomatoclastes oppose en fait deux visions de la langue et du nom (dont le Nom de Dieu est l'emblème suprême), que l'on peut caractériser comme une vision mystique et une approche rationnelle. D'un côté, les onomatoclastes comprennent le nom comme un signe conventionnel, signe humain, qui donc ne peut avoir aucune prétention en tant qu'instrument de connaissance de Dieu, car Dieu, dans Son Être, est ineffable. D'un autre côté, les onomatodoxes défendent une conception ontologique du nom, qui apparaît au contraire comme un signe épiphanique, une présence de la réalité nommée, donc un moyen d'accès à l'être. Prenant part à cette querelle qui rencontre ses propres préoccupations spirituelles, le père Pavel Florenskij va prendre la défense de l'onomatodoxie.

La crise du mont Athos sera donc très féconde intellectuellement: elle est l'occasion d'une actualisation de la querelle médiévale opposant réalisme et nominalisme; elle sera aussi à la source d'une réappropriation de la pensée

\footnotetext{
4. Na gorax Kavkaza.

5. La prière de Jésus est une sorte de prière du publicain (Luc 18, 13) qui s'appuierait sur le culte paulinien du Nom de Jésus: «Господи Иисусе Христе, Сыне Божий, помилуй мя грешного » («Seigneur Jésus-Christ, Fils de Dieu, aie pitié de moi, pécheur»).

6. «Имя Божие - Сам Бог, Сам Бог, Сам Бог !».
} 
des énergies de Grégoire Palamas, qui permet de comprendre le Nom de Dieu comme une énergie divine donnant accès à Dieu lui-même dans la prière fervente. Depuis les années 1990, de nombreux travaux scientifiques ont été consacrés à la question de l'onomatodoxie ainsi qu'à l'éminent apport du père Florenskij à ce débat. En ce qui concerne les publications en langue française, il faut bien sûr citer l'article de Maryse Dennes, «Les glorificateurs du nom: une rencontre de l'hésychasme et de la philosophie au début du XXe siècle en Russie » ${ }^{7}$, dans lequel elle souligne notamment la dimension gnoséologique du Nom de Dieu dans la définition qu'elle donne de la prière de Jésus: «La répétition continuelle du Nom de Jésus devait conduire progressivement à l'état d'illumination intérieure et de fusion avec Dieu ${ }^{8}$. C'est bien cette dimension gnoséologique qui sera au cœur de la réflexion du père Florenskij dans son ouvrage Les noms.

Il est à remarquer que la réflexion théorique du père Florenskij sera toujours accompagnée d'une réflexion pratique, fruit de l'observation et de la méditation des prénoms de son entourage. Et c'est cette dimension pratique de sa pensée qui constituera la deuxième partie de son ouvrage Les noms, qui se présente ouvertement comme un dictionnaire des prénoms (slovar' imen). Or, cette corrélation entre théorie et pratique, concernant la question du nom (imja), rencontre la réflexion menée au même moment par les poètes symbolistes et post-symbolistes. En effet, les deux premières décennies du $\mathrm{XX}^{\mathrm{e}}$ siècle, temps de la modernité auquel on a donné le nom d'Âge d'argent, période de renouveau spirituel et d'effervescence intellectuelle et artistique, se caractérisent notamment par un brouillage des limites qui séparaient traditionnellement les différents domaines de la pensée. L'onomatologie est ainsi tout à la fois un objet de réflexion pour les linguistes, les philosophes, les théologiens et les poètes, et la réflexion de Florenskij se trouve justement à la croisée de toutes ces disciplines.

Un des fondements de la modernité poétique est la mise en question de la nature propre de chaque art: les poètes de l'Âge d'argent vont ainsi exposer la question de la nature du nom poétique, dans leur pratique poétique même, souvent largement métapoétique, mais aussi dans de nombreux essais théoriques, qui parfois tendent à prendre plus de place que leur pratique même. Ainsi, par exemple, la question de la nomination est au centre de l'essai du poète symboliste Andrej Belyj «La magie des mots » 9 , daté de 1910, qui affirme dès l'introduction: «La langue est le plus

7. M. Dennes, «Les glorificateurs du nom: une rencontre de l'hésychasme et de la philosophie au début du XXe siècle en Russie», Slavica occitania [Toulouse], nº 8, 1999, p. 143-171.

8. Ibid., p. 144-145.

9. A. Belyj, «Magija slov», in Simvolizm kak miroponimanie, Moskva, Respublika, 1994. 
puissant instrument de création. Lorsque je nomme un objet, j'affirme son existence. Toute connaissance découle de la nomination ${ }^{10}$. De même, on retrouve cette même affirmation de la corrélation entre le nom et la connaissance dans l'essai «De la nature du verbe» de Mandel'štam, daté de 1922, signe que la question de l'onomatologie dépasse les querelles d'école entre symbolistes et post-symbolistes: «Le nom est déjà définition, déjà "nous savons quelque chose" ${ }^{11}$.

On retrouve encore ici la dimension gnoséologique du nom, qui est aussi un des motifs récurrents des Noms du père Pavel Florenskij. Nous verrons à présent la spécificité de la pensée du père Pavel Florenskij dans son ouvrage Les noms, qui concerne cette fois les noms propres, et nous chercherons à dégager la méthode selon laquelle sa réflexion se construit.

\section{Méthode du père Pavel Florenskij dans Les noms}

Le rapport entre le nom et la connaissance de l'être personnel est au cœur de l'ouvrage Les noms. Le père Florenskij cherche à donner un cadre rationnel à ce qui apparaît comme une intuition poétique dans les écrits des poètes. Il écrit par exemple: «Il n’y a pas de doute: dans la création littéraire, les noms sont des catégories de connaissance de la personne, parce que dans l'imagination créatrice, ils ont la force de formes personnelles. Cependant il en est ainsi non pas seulement dans les œuvres de la création individuelle, mais aussi dans la création populaire ${ }^{12}$. On remarque ici l'emploi de concepts appartenant à l'histoire de la philosophie (catégorie, forme), qui encadrent l'expression d' "imagination créatrice» caractéristique de la pensée poétique. En effet, l'onomatologie, comme l'indique le sous-titre de l'œuvre, est vue par le père Pavel Florenskij comme une science qui prend place dans le vaste projet d'anthropodicée de l'auteur.

Mais la citation nous indique également que le point de départ méthodologique de l'auteur est bien la création littéraire; c'est dans la littérature que le père Florenskij va chercher les preuves de sa démonstration. On observe ainsi continuellement dans l'œuvre un mouvement de va-et-vient

10. A. Belyj, «Magija slov», p. 131: « Язык - наиболее могущественное орудие творчества. Когда я называю словом предмет, я утверждаю его существование. Всякое познание вытекает уже из названия ».

11. O. Mandel'štam, «O prirode slova», in Sobranie sočinenij, G. Struve, B. Filippova (dir.), Moskva, Terra, 1993, 4 vol., t. II, p. 249: «Имя уже определение уже “что-то знаем” ».

12. P. Florenskij, Imena, p. 184: «Нет сомнения: в литературном творчестве имена суть категории познания личности, потому что в творческом воображении имеют силу личностных форм. Однако это так - не только в произведениях индивидуального творчества, но и в творчестве народном». 
entre des passages relevant d'une lecture attentive de la création poétique, que le père Florenskij s'approprie, et des passages répondant à une logique discursive, dans lesquels la pensée s'exprime au moyen de concepts philosophiques. On a souvent parlé de l'influence de la pensée philosophique (ou théologique) sur la création poétique: c'est dans ce sens, par exemple, que Maryse Dennes, dans l'article cité plus haut, commente le poème de Mandel'štam «Et maintenant sur l'Athos... ${ }^{13}$, que l'on pourrait lire comme une justification poétique de l'onomatodoxie ${ }^{14}$. L'ouvrage Les noms constitue au contraire un exemple éloquent de l'influence de la pensée poétique sur la réflexion philosophique. En effet, le père Pavel Florenskij fait sienne l'intuition poétique des créateurs, unifiant création individuelle et création collective, populaire, comme l'indique la citation proposée. Par cet ouvrage réflexif qui se présente comme scientifique, il affermit donc paradoxalement la légitimité de la parole poétique, et conforte le mythe de la vérité poétique, déjà bien ancré à l'Âge d’argent.

L'ouvrage s'ouvre ainsi sur différentes citations de Mérimée, Balzac ou Hugo soulignant le poids à la fois sémantique et existentiel des noms propres, et le père Florenskij conclut: «le nom doit être une image. Un poète qui ne sait pas cela ne sait rien ${ }^{15}$. Cette assertion définitive montre la nécessité vitale du propos pour son auteur. Au chapitre suivant, le père Florenskij se tourne vers la littérature russe, et vers le premier poète entre tous, Pouchkine. Il étudie ainsi longuement le nom de Marioula, hérö̈ne du poème narratif Les Tsiganes, dont il dit que "sa matière sonore informe tout le poème» ${ }^{16}$. Il évoque alors la métaphysique des sons; «pour faciliter l'analyse ${ }^{17}$, transcrit le nom Marioula en hébreu, et par souci d'impartialité, utilise le système de caractérisation des sons de Fabre d'Olivet ${ }^{18}$, puis l' «alphabet organique» de Brosses ${ }^{19}$. Ce long exposé se résume enfin à l'analyse du son «ou », interprété ultimement comme le motif de la force élémentaire féminine située à la

13. O. Mandel’štam, «I ponyne na Afone... » [1915], in Sobranie sočinenij, t. I, p. 45.

14. Voir à ce sujet F. Corrado-Kazanski, Verbe et poésie dans la Russie de l'Âge d'argent. Le statut de la langue, du nom et du verbe dans la poésie, la philosophie et la théologie russes au début du vingtième siècle, Sarrebruck, Éditions universitaires européennes, 2010, p. 59-62.

15. P. Florenskij, Imena, p. 172: «Имя должно быть образом. Поэт, который не знает этого, ничего не знает».

16. Ibid., p. 174: «Это “глубоко женственное и музыкальное имя” есть звуковая материя, из которой оформливается вся поэма».

17. Ibid., p. 176 .

18. Le père Florenskij donne en note la référence française suivante: A. Fabre d'Olivet, $L a$ langue hébräque restituée et le véritable sens de mots hébreux, Paris, Barrois, 1815.

19. Le père Florenskij donne en note la référence française suivante: C. de Brosses, Traité de la formation mécanique des langues, Paris, Saillant, 1765, suivie de sa traduction russe: де Броссе Ш., Рассуждение о механическом составе языков и физических началах этимологии, перевод А. Никольского, Санкт-Петербург, 1821-1822. 
limite de l'être et du non-être... Cette étude des sons du nom de Marioula constitue la première analyse onomatologique de l'œuvre, dans la tradition du Cratyle de Platon. Il faut noter cependant que le père Florenskij ne cite pas expressément le Cratyle, à la différence du père Bulgakov qui lui consacre une annexe dans son ouvrage La philosophie du nom ${ }^{20}$. C'est peut-être qu'en onomatologie, il préfère la filiation des poètes à celle des philosophes...

Comme un poète, en effet, le père Pavel Florenskij reconnaît au nom son éminent statut de matière sonore qui fait sens (ou qui est sens). Il écrit plus loin: "De même que le nom s'incarne dans le son, son essence spirituelle est accessible avant tout par le ressenti de sa chair sonore ${ }^{21}$. Il dévoile ainsi la méthode selon laquelle il étudie les noms: il s'agit avant tout de méditer la matière sonore du nom, et c'est de cette méditation que naîtra l'intuition du sens et de l'être, la connaissance de la personne. Ici, la méthode du père Florenskij rencontre la pratique des poètes, qui sont guidés dans l'acte de nomination poétique par la matière sonore de la langue, ainsi que la pratique des hommes de prières, en particulier des onomatodoxes, pour qui la répétition du Nom de Jésus, dans sa matérialité sonore où Dieu s'incarne, est une porte d'entrée à l'expérience mystique.

Tout en soulignant les points de contact entre onomatologie poétique et onomatologie philosophique (et implicitement, théologique), le père Florenskij cherche à asseoir l'indépendance de ce qu'il considère comme une science, et qui sera une science hybride: «[...] l'onomatologie doit se tenir de manière indépendante, en partie fondée sur des preuves philosophiques, en partie également sur des observations et des conclusions tirées de l'expérience historique ${ }^{22}$. Cette affirmation paradoxale ne fait que révéler encore davantage la nature hybride, voire baroque, du mode de pensée du père Florenskij ${ }^{23}$. Sa réflexion sur les noms se trouve à la croisée des voies intellectuelles poétiques, théologiques, philosophiques; elle relève à la fois du raisonnement philosophique et de l'observation empirico-poétique. L'ouvrage même se compose de deux parties: la première, théorique, est consacrée à un exposé de la notion de nom tel que l'entend le père Florenskij, la seconde, pratique, constitue un dictionnaire

20. S. Bulgakov, Filosofija imeni, Moskva, Iskusstvo / Sankt Peterburg, Inapress, 1999, p. 233-238.

21. P. Florenskij, Imena, p. 175: «Но как имя воплощено в звуке, то и духовная сущность его постигается преимущественно вчувствованием в звуковую его плоть ».

22. Ibid., p. 186: «[...] ономатологии надлежит держаться самостоятельно, частью на философских доказательствах, частью же наблюдениями и наведениями исторического опыта».

23. Voir à ce sujet F. Corrado-Kazanski, «Logos et baroque à l'Âge d'argent», Modernités russes [Centre d'études slaves André Lirondelle, université Jean Moulin Lyon 3], n 11, 2011, L'unité sémantique de l'Âge d'argent, p. 65-75. 
des prénoms. Nous présenterons les définitions du nom proposées par l'auteur avant d'étudier la composition d'une de ses méditations sur un nom qui forment les articles de ce dictionnaire.

\section{Les définitions du nom}

Pour déterminer les liens entre nom et personne, le père Florenskij use de différents concepts relevant de différentes traditions de pensée. Avant tout, il justifie sa position de manière étymologique, ce qui est un procédé fréquent dans son mode de pensée. Il rappelle ainsi qu'en grec hellénistique, et en particulier en grec biblique, comme en hébreu, le mot óvoua peut justement signifier la personne (lico). Les notions de personne (lico et ličnost', l'un plus concret, l'autre plus abstrait) apparaissent ainsi comme des développements de la notion originelle de nom (imja), que le père Florenskij va s'attacher à expliciter au moyen de différentes définitions. Il emploie tout d'abord le concept de forme, déjà évoqué ${ }^{24}$, parfois déployé en la notion de formule.

L'expérience historique doit montrer si le peuple, si la langue même prend les noms pour de vides sobriquets, arbitrairement unis aux personnes qui les portent, et qui par conséquent n'apportent rien à la connaissance de ces personnes, ou bien au contraire s'il faut trouver dans le nom la Formule de la personne, la clef de l'organisation et la construction de sa figure personnelle, une sorte d'universale, tout à fait concret, tout à fait proche de l'haecceitas de l'homme, bien qu'il ne se confonde pas à elle ${ }^{25}$.

Ici encore apparaît nettement la nature baroque de la pensée du père Florenskij, qui mêle des concepts appartenant à différentes traditions spéculatives: la notion de formule évoque la pensée magique, les recherches théosophiques, tandis que la référence aux notions latines continue la querelle médiévale des universaux, et que le contenu même de la proposition renvoie aussi au débat opposant onomatodoxes et onomatoclastes. La notion de forme, développée en «formule», qui montre la parenté de

24. Voir la citation donnée en note 12.

25. P. Florenskij, Imena, p. 184: «Исторический опыт должен показать, признает ли народ, признает ли самый язык имена пустыми кличками, условно присоединенными к их носителям и потому ничего не дающими познанию носителя, или же пологает найти в имени Формулу личности, ключ к вскладу и строению личного облика, некоторое universale, весьма конктретное, весьма близкое к этости, haecceitas человека, хотя с этостью и не тождественное». L'haecceitas ou «eccéité» correspond au tode ti d'Aristote; le terme a été créé par Duns Scot. Ipseitas peut être considéré comme synonyme; c’est ce par quoi un individu se distingue des autres. 
la pensée de Florenskij avec la pensée magique, est ailleurs associée à celle de force, ou d'énergie, ce qui souligne en même temps l'appartenance de l'auteur au courant de la pensée mystique orthodoxe. Il parle en effet plus loin de «la compréhension des noms comme forces formantes» (ponimanie imen kak formoobrazujuščix sil) ${ }^{26}$, ou encore de "forces substantielles » (substancial'nye sily), ou "substances potentielles» (silovye substancii), ou «énergies» (ènergii) ${ }^{27}$. Plus loin encore, il adjoint au concept de forme celui de type: «Par le nom s'exprime ainsi le type de la personne, sa forme ontologique, qui détermine le devenir de sa construction spirituelle et psychique ${ }^{28}$. La notion de type est aussi redoublée par celle d'invariant: «Il faut appréhender les noms comme des invariants de la personne ${ }^{29}$, ou encore par la notion platonicienne d'idée, par exemple dans la citation suivante:

Le nom est considéré par l'Église, et à sa suite par tout le peuple orthodoxe, comme le type, comme la norme spirituelle concrète de l'être personnel, comme l'idée, et le saint est considéré comme sa meilleure expression, ayant rendu son existence empirique transparente, afin qu'à travers lui brille la lumière très noble du dit nom. Mais le nom est ontologiquement premier, et la personne qui le porte, fût-elle sainte, est seconde ${ }^{30}$.

Toutes ces différentes tentatives de définitions, toutes ces variations sur le même thème de la définition du nom montrent que le père Florenskij ne mène pas une démonstration au sens logique, traditionnel du terme. Partant d'observations onomastiques tirées de la littérature (concernant essentiellement le nom en tant qu'orientation de la destinée d'un personnage), le père Florenskij cherche à donner une forme logique, discursive, à cette intuition qu'il partage avec les poètes, à savoir celle du nom comme figure, révélation de l'être, moyen d'accès à la connaissance. Sûr qu'il y a dans le nom "quelque chose qui échappe à l'analyse rationnelle» (čto-to uskol'zajuščee ot racionalističeskogo analiza) ${ }^{31}$, le père Florenskij se libère à son tour des formes strictes de la rationalité pour mieux coller à ce quelque

26. P. Florenskij, Imena, p. 189.

27. Ibid., p. 196.

28. Ibid., p. 211: «Итак, именем выражается тип личности, онтологическая форма ее, которая определяет далее ее духовное и душевное строение».

29. Ibid., p. 214: «Имена и должны быть рассматриваемы как такие инварианты личности ».

30. Ibid., p. 188: « Имя оценивается Церковью, а за нею - и всем православным народом, как тип, как духовная конкретная норма личностного бытия, как идея, а святой - как наилучший ее выразитель, свое эмпирическое существование соделавший прозрачным так, что через него нам светит благороднейший свет данного имени. И все-таки имя - онтологически первое, а носитель его, хотя бы и святой, - второе».

31. Ibid., p. 205. 
chose qui ne peut relever que de l'expérience poétique ou de l'expérience mystique. De ce point de vue, ces multiples définitions du nom, selon différentes terminologies, révèlent aussi le vain effort du logos humain à rendre compte d'une réalité qui le dépasse, puisqu'il s'agit en fin de compte de reconnaître la primauté et la puissance du nom en tant que tel.

La dernière citation montre en effet que l'être contenu en puissance dans le nom peut se déployer de différentes manières, depuis un pôle positif occupé par le saint, jusqu'au pôle négatif occupé par le damné. Ces deux pôles tracent ainsi tout l'espace de la liberté de la personne, à l'intérieur des limites posées par le nom. Florenskij n'élude donc pas la question de la liberté, dans toute sa dimension chrétienne existentielle.

Le nom prédétermine la personne et indique les limites idéales de sa vie. Mais cela ne signifie pas que, déterminée par son nom, la personne n'est pas libre dans son nom - à l'intérieur de ses limites. Et avant tout, tout nom donné est un spectre entier de prédéterminations morales et un faisceau de différents chemins de vie. Le pôle supérieur du nom est le pur rayon individuel de la lumière divine, le prototype de la perfection scintillant dans la figure de saint du dit nom. Le pôle inférieur de ce même nom s'enfonce dans la géhenne, comme la totale perversion de la vérité divine du dit nom, mais qui y reste invariant ${ }^{32}$.

On comprend ici que tout nom, dans sa manifestation supérieure, se rend lui-même à son tour transparent au «Nom qui est au-dessus de tout nom ${ }^{33}$, le Nom de Jésus, seul vrai «prototype de la perfection», en qui seul coïncident le nom (Jésus, qui signifie «Dieu sauve " ${ }^{34}$ ) et la personne, le Sauveur du monde. La réflexion sur les noms humains ne prend tout son sens que dans le cadre de l'ontologie chrétienne qui repose sur l'incarnation de Dieu, et l'incarnation du Nom. Essentiel est le symbole de la lumière, présent dans les deux dernières citations, mais également sous-entendu dans la définition même du nom comme énergie de l'être personnel. Là encore, la lumière du nom est le reflet de la Lumière sans déclin qu'est le

32. Ibid., p. 225: «Имя предопределяет личность и намечает идеальные границы ее жизни. Но это не значит,что именем определенная, личность несвободна в своем имени - в его пределах. И прежде всего: каждое данное имя есть целый спектр нравственных самоопределений и пучок различных жизненных путей. Верхний полюс имени - чистый инднвидуальный луч божественного света, первообраз совершентсва, мерцающий в святом данного имени. Нижний полюс того же имени уходит в геенну, как полное извращение божественной истины данного имени, но тут и остается инвариантным ».

33. Épitre aux Philippiens 2, 9.

34. Voir Évangile selon saint Matthieu 1, 21: «elle enfantera un fils auquel tu donneras le nom de Jésus, car c'est lui qui sauvera son peuple de ses péchés». 
Christ. C'est parce que le nom et la lumière qui le traverse renvoient au Nom de Jésus, garantissant ainsi l'enracinement de l'être personnel dans le Christ ${ }^{35}$, que le nom garantit l'intégrité de la personne, qu'il est «ce qui tient» la personne. On comprend alors la nécessité ontologique de ces travaux sur les noms pour l'homme de Dieu qu'est le père Florenskij, puisqu'ils touchent à la fois au cœur de Dieu et au cœur des hommes. On comprend également l'importance, en termes d'expérience spirituelle, de la méditation qu'il fait sur son propre nom, Pavel, que nous allons à présent commenter.

\section{Le nom de Pavel (Paul)}

Le dictionnaire des prénoms que propose le père Florenskij dans la seconde partie de son ouvrage est composé de dix-huit chapitres consacrés aux prénoms russes les plus fréquents et les plus représentatifs. Ils sont souvent présentés par couples masculin-féminin. Sont ainsi commentés les prénoms suivants: Aleksandr, Aleksandra, Aleksej, Anna, Vassilij, Sofija, Vladimir, Ol'ga, Konstantin, Elena, Nikolaj, Ekaterina, Dmitrij, Varvara, Pavel, Ljudmila, Vera, Mixail. Le plus souvent, le chapitre s'ouvre sur l'écoute des sonorités du nom, ou sur une étude étymologique du nom, qu'elle relève d'une étymologie scientifique ou populaire, en fonction de ce qui est le plus significatif aux yeux de l'auteur. La méditation sur le son ou le sens du nom révèle ensuite progressivement l'être du nom en tant que forme de la personne. Les figures de saints portant ce nom sont souvent évoquées, en tant qu'elles font le plus clairement transparaitre le type originel du nom. Il est à noter que le chapitre consacré au nom de Paul est beaucoup plus long que les autres, comme si, dans un jeu de la subjectivité et de l'objectivité, une méditation approfondie sur son propre nom était gage de lucidité spirituelle concernant les autres noms.

La méditation sur le nom de Pavel commence immédiatement par l'évocation de l'apôtre Paul, qui domine selon l'auteur toutes les représentations du nom. Le père Florenskij évoque le double nom Saul-Paul. C'est dans l'étymologie du nom hébreu Saul, originellement Saoul, ou Shaoul, que le père Florenskij perçoit l'être de la personne, configuré par la notion de désir, ou volonté (xotenie) présente dans le nom. Saul est à la fois le désirant et le désiré. Si la volonté, dans sa dimension ontologique, est la caractéristique du prénom, elle sera aussi la caractéristique de la personne. Le père Florenskij écrit:

35. Voir Évangile selon saint Jean 15, 4: «Demeurez en moi comme je demeure en vous!». 
En Paul, cette pression de la volonté, bien qu'insignifiante en elle-même, s'avère être la principale caractéristique de la composition du nom, et tout le reste est secondaire et dérivé, voire simplement fortuit. Ainsi le caractère de Paul doit-il être compris comme communiquant très facilement à l'origine de l'être ${ }^{36}$.

Cette «pression de la volonté» est aussi nommée «force élémentaire» (ou "pression élémentaire», stixijnyj napor). Paul est plus loin défini comme "volonté pure» (čistaja volja) tendant vers la «forme parfaite, l'idée platonicienne, la spiritualité non pas en tant que pensée abstraite, quoique vraie, mais existentiellement impuissante, mais en tant que force ${ }^{37}$, qui n'est autre que la personne même du Christ Sauveur. Suit une méditation sur la quête de l'incarnation, caractéristique de Paul selon le père Florenskij, dans laquelle on est tenté de voir une sorte de confession spirituelle, à cause notamment de la force des images convoquées : «Le Christ est ressenti par lui [Paul] comme un courant de feu pénétrant le Cosmos et le pénétrant lui-même en partie ${ }^{38}$. C'est ainsi que le père Florenskij voit en la volonté et la foi les deux pôles entre lesquels se construit la personne de Paul. Pour appuyer son argumentation reposant jusqu'alors sur l'intuition donnée par la méditation, le père Florenskij fait référence au canon des icônes de saint Paul, où prédomine le ton vert, «signe de la force élémentaire qui se trouve au fondement de la vie», et l'or, «le Logos, le Christ, la grâce, la foi » ${ }^{39}$. La pensée symbolique de l'auteur se trouve donc confortée par la symbolique des couleurs de l'icône. La nécessité du symbole est aussi implicitement évoquée dans la longue partie consacrée à la raison de Paul (razum), secondaire par rapport à la volonté, néanmoins longuement définie comme "pensée dialectique» (dialektičeskoe myšlenie) ${ }^{40}$, mouvante, contradictoire, en prise avec la réalité, cherchant à «exprimer par le verbe la volonté anté-verbale et supra-verbale ${ }^{41}$. Le texte devient ici autobiographie intellectuelle qui

36. P. Florenskij, Imena, p. 310: « В Павле этот напор воли, хотя бы и ничтожный сам по себе, все-таки берется как главное характерное в строение имени, а всему остальному дается цена вторичного и производного, если не просто случайного. Таким образом, характер Павел следует понимать как весьма легко сообщающийся с первоосновой бытия ".

37. Ibid., p. 312: «Предмет его томления - совершенная форма, Платоновская идея, духовность, но не как отвлеченная мысль, т.е. хотя истинная,но бытийственнобессильная, а как сила».

38. Ibid., p. 313: «Христос ощущается им как огненная струя, пронизывающая Космос и его самого, в частности».

39. Ibid., p. 316: «зеленые цвета [...] знаменуют стихийные основы мировой жизни»; «золото же - [...] Логос, Христос, благодать, вера».

40. Ibid., p. 317 .

41. Ibid.: «П опытка выразить словом до-словесную и сверх-словесную волю». 
peut se lire comme une auto-justification de la forme que prend la pensée du père Florenskij, entièrement sous le signe du symbole et de l'antinomie. Enfin, le père Florenskij conclut sa méditation sur le nom de Paul par la troisième composante du nom et de la personne, après la volonté et la foi : la souffrance, qui provient de la nature tragique de l'antinomie, que celle-ci ait lieu à l'intérieur de la personne ou entre la personne et le monde. Cette conclusion ne peut que résonner comme une prophétie de la part de celui qui mourut en martyr, victime en 1937 de la persécution stalinienne.

En conclusion, nous pouvons dire que la méditation sur les noms du père Florenskij, visant à révéler la structure du nom et de la personne, se situe à un haut niveau d'abstraction, conformément à la visée de l'idée qu'il poursuit, tout en étant profondément ancrée dans le réel, fidèle à une vision symbolique du monde qui réunit le monde visible et le monde invisible. Dans son ouvrage La pensée et la langue, le père Florenskij écrit:

C'est que le verbe, en tant que médiateur entre le monde intérieur et le monde extérieur, c'est-à-dire, en tant qu'amphibie vivant ici et là-bas, installe évidemment des sortes de fils entre l'un et l'autre monde, et ces fils, si peu visibles soient-ils pour l'œil d'un positiviste, sont bien ce pour quoi existe le verbe lui-même, ou tout du moins sont-ils le fondement premier de toutes les autres fonctions du verbe ${ }^{42}$.

Pour le père Florenskij, le nom est bien ce fil reliant la personne qui le porte à l'Idée qui constitue son prototype. Ultimement, le nom relie la personne au Nom des noms, le Nom de Jésus; il réalise donc, dans sa manifestation la plus sainte, l'unité de toute personne avec la personne du Christ. La pensée du nom est donc partie intégrante de la théologie de l'incarnation; elle contribue à fonder l'anthropologie chrétienne.

Florence CORRADO-KAZANSKI

Université Michel de Montaigne - Bordeaux 3

FRE 3392

42. P. Florenskij, U vodorazdelov mysli, IV. Mysl’ i jazyk, in Sočinenija, t. 3(1), p. 230 : «Дело в том, что слово, как посредник между миром внутренним и миром внешним, т.е. будучи амфибией, живущей и там и тут, устанавливает, очевидно, нити своего рода между тем и другим миром, и нити эти, какими бы ни были они мало приметными взору позитивиста, суть, однако, то, ради чего существует самое слово, или по крайней мере суть перво-основа всех дальнейших функций слова». 